\title{
Effectiveness of expiratory technique and induced sputum in obtaining good quality sputum from patients acutely hospitalized with suspected lower respiratory tract infection: a statistical analysis plan for a randomized controlled trial
}

Mariana Bichuette Cartuliares ${ }^{1,2^{*}}$ (D) , Helene Skjøt-Arkil ${ }^{1,2}$, Flemming Schønning Rosenvinge ${ }^{4}$, Christian Backer Mogensen ${ }^{1,2}$, Thor Aage Skovsted ${ }^{3}$ and Andreas Kristian Pedersen ${ }^{2,5}$

\begin{abstract}
Background: Targeted antimicrobial treatment is essential to avoid unnecessary use of broad-spectrum antibiotics and antimicrobial resistance. Targeted treatment relies on a precise microbiological diagnosis - in pneumonia, this poses a challenge as the usefulness of Gram stains and cultures is highly dependent on the quality of the sputum sample.

This study aims to examine adverse effects and quality of sputum samples obtained by expiratory techniques (forced expiratory technique and sputum induction) compared with tracheal suction. The hypothesis is that expiratory techniques are non-inferior to tracheal suction in obtaining samples from the lower respiratory tract. This statistical analysis plan (SAP) describes the study design, method, and data analysis of the trial to increase transparency, avoid reporting bias or data-driven analysis and increase the study's reproducibility.

Method: The design is a pragmatic, non-inferiority, parallel-arm randomized controlled trial including 280 patients admitted with suspected lower respiratory infection to two emergency departments. Patients are randomized to a usual care group, where sputum samples are collected by tracheal suction or to an intervention group where sputum samples are collected by forced expiratory technique and sputum induction. The statistical analysis will follow an intention-to-treat protocol. This SAP is developed and submitted before the end of recruitment, database closure, and statistical analyses.
\end{abstract}

Discussion: The results of this study will provide valuable knowledge to clinical practice by comparing adverse effects and sputum sample quality associated with different sample methods.

\footnotetext{
* Correspondence: mariana.cartuliares@rsyd.dk

'Emergency Department, University Hospital of Southern Denmark, Kresten Philipsens vej 15, 6200 Aabenraa, Denmark

${ }^{2}$ Department of Regional Health Research, University of Southern Denmark, Aabenraa, Denmark

Full list of author information is available at the end of the article
}

(c) The Author(s). 2021 Open Access This article is licensed under a Creative Commons Attribution 4.0 International License, which permits use, sharing, adaptation, distribution and reproduction in any medium or format, as long as you give appropriate credit to the original author(s) and the source, provide a link to the Creative Commons licence, and indicate if changes were made. The images or other third party material in this article are included in the article's Creative Commons licence, unless indicated otherwise in a credit line to the material. If material is not included in the article's Creative Commons licence and your intended use is not permitted by statutory regulation or exceeds the permitted use, you will need to obtain permission directly from the copyright holder. To view a copy of this licence, visit http://creativecommons.org/licenses/by/4.0/. The Creative Commons Public Domain Dedication waiver (http://creativecommons.org/publicdomain/zero/1.0/) applies to the data made available in this article, unless otherwise stated in a credit line to the data. 
Trial registration: Clinicaltrials.gov, NCT04595526. Submitted on October 19, 2020

Keywords: Randomized controlled trial, Statistical analysis plan, Sputum sample, Tracheal suction, Forced expiratory technique, Induced sputum, Respiratory tract infection

\section{Administrative information $\{1\}$}

Note: the numbers in curly brackets in this protocol refer to recommended guidelines for the content of a statistical analysis plan of a clinical trial developed by Gamble et al. [1] (Additional file 6). The protocol has been reported according to SPIRIT reporting guidelines [2].

\begin{tabular}{|c|c|}
\hline Full study title $\{1 a\}$ & $\begin{array}{l}\text { Effectiveness of expiratory technique } \\
\text { and induced sputum in obtaining } \\
\text { good quality sputum from patients } \\
\text { acutely hospitalized with suspected } \\
\text { lower respiratory tract infection: a } \\
\text { statistical analysis plan for a } \\
\text { randomized controlled trial }\end{array}$ \\
\hline $\begin{array}{l}\text { Clinicaltrials.gov number } \\
\{1 b\}\end{array}$ & $\begin{array}{l}\text { NCT04595526 Registered on 10th October } \\
2020\end{array}$ \\
\hline Ethics Committee number & $\begin{array}{l}\text { S-20200133 Registered on 22th } \\
\text { September } 2020\end{array}$ \\
\hline $\begin{array}{l}\text { The Danish Data } \\
\text { protecting Agency } \\
\text { number }\end{array}$ & $\begin{array}{l}\text { 20/41767 Registered on 11th September } \\
2020\end{array}$ \\
\hline SAP version $\{2\}$ & Version 1.0 date 18.12 .2020 \\
\hline
\end{tabular}

Protocol version $\{3\} \quad$ Ethics Committee protocol version 4.0 date 26.01.2021

SAP revision $\{4 \mathrm{a}\}$

No SAP revisions. Protocol revised 1st of December 2020

Deviations from the protocol are described with justifications in section $\{19 \mathrm{C}\}$

Roles and responsibility \{5-\}

SAP author $\{6 a\}$

Statistician responsible $\{6 b\}$

Clinical lead $\{6 c\}$

Collaborators/coming manuscript authors:
Administrative information $\{1\}$ (Continued)

\begin{tabular}{ll} 
Full study title \{1a\} $\quad \begin{array}{l}\text { Effectiveness of expiratory technique } \\
\text { and induced sputum in obtaining } \\
\text { good quality sputum from patients } \\
\text { acutely hospitalized with suspected } \\
\text { lower respiratory tract infection: a } \\
\text { statistical analysis plan for a } \\
\text { randomized controlled trial }\end{array}$ \\
\hline Odense University Hospital, Denmark \\
Christian Backer Mogensen, MD, Ph.D., \\
professor \\
Emergency Department, University \\
Hospital of Southern Denmark, and \\
Department of Regional Health Research, \\
Faculty of Health Sciences, University Of \\
Southern Denmark \\
Thor Aage Skovsted, MD, Ph.D. \\
Department of Biochemistry and \\
Immunology, University Hospital of \\
Southern Denmark, Denmark
\end{tabular}

\section{Background \{7\}}

Lower respiratory tract infection (LRTI) is a serious condition associated with high mortality, morbidity and economic burden [3-5]. Appropriate and targeted antimicrobial treatment is vital to avoid unnecessary use of broad-spectrum antibiotics and subsequent development of bacterial resistance.

Sputum Gram stain and culture from patients with LRTI are important when selecting a targeted antimicrobial treatment $[6,7]$.

Several clinical limitations interfere with the practice of obtaining a representative specimen from the lower respiratory tract (LRT). Many patients find it difficult to expectorate, sputum is often contaminated by oropharyngeal microbiota, and some patients have already been treated with antibiotics at admission, compromising the results from sputum culture [8-10]. The guidelines from the American Thoracic Society and Infectious Diseases Society of America recognize the challenges in obtaining a valid sputum sample. They recommend sputum analyses based on individual clinical assessment, local etiological considerations, and local antimicrobial stewardship [11].

The Danish guidelines [12] follow the National Institute for Health and Care Excellence guidelines [13] and are further developed by experts in the fields of microbiology, infectious disease, and emergency medicine. It is required that sputum samples must be collected at arrival at the emergency department (ED) from all patients admitted with suspected LRTI, and advocate tracheal suction as an 
optimal collecting method [12]. Despite indications from some studies that tracheal suction might be superior to expectorated sputa in diagnosing LRTI [14, 15], low accuracy and misclassification of poor samples have been reported $[16,17]$. Tracheal suction is widely performed to clear pulmonary secretions in mechanically ventilated patients [18] but reported as a painful experience for the patient [19] being associated with adverse events such as hypoxia, oxygen desaturation, and mucosal bleeding [20]. Forced expiratory technique and sputum induction are considered safe and straightforward methods [21] and are used successfully to clear airways and increase sputum production, facilitating collection for microbiological analyses when patients are unable to expectorate [22-25].

The most effective method to collect a representative specimen from the LRT remains uncertain. The efficacy of tracheal suction, forced expiratory technique and sputum induction to obtain sputum samples of high quality has not been investigated in an ED context.

\section{Hypothesis, aim, and objectives $\{8+12\}$}

We hypothesize that expiratory techniques (forced expiratory technique and sputum induction) are noninferior to tracheal suction in obtaining sputum samples from the lower respiratory tract.

This study aims to investigate the effectiveness of expiratory techniques compared to tracheal suction on the quality of sputum samples collected from patients acutely hospitalized with suspected LRTI.

The following objectives will be explored:

1. Is the proportion of suitable (good quality) sputum samples different when comparing samples obtained by tracheal suction to samples obtained by expiratory techniques?

2. Is the number of adverse events different when comparing tracheal suction to expiratory techniques?

3. How do patients experience the two sputum sample collecting procedures?

This statistical analysis plan (SAP) will describe the statistical analysis to be executed on primary and secondary outcomes to increase study transparency, reproducibility, and validity. This SAP was developed after the study protocol was registered with the following organizations: Danish ethics committee; clinical trials; data protection agency and, before database closure, data review; and commencement of any statistical analyses.

\section{Method}

This SAP followed the recommended guidelines for a statistical analysis plan of a clinical trial developed by
Gamble et al. [1]. A checklist for SPIRIT reporting guidelines and a template for the schedule of enrolment [2] is attached as Additional files 1 and 3. The study will follow the Consolidation Standard of Reporting Trials (CONSORT) guidelines (parallel-group randomized trials) [26].

\section{Study design and setting $\{9\}$}

The study design is a pragmatic, non-inferiority, randomized controlled trial (RCT). The trial is conducted from the 9th of November 2020 until the 1st of July 2021 at the University Hospital of Southern Denmark including the hospitals of Aabenraa and Sønderborg with a hospital coverage of approximately 250,000 inhabitants. The standard method for sputum collection at both EDs is tracheal suction.

Six project assistants from the ED (experienced nurses and physiotherapist) will identify eligible patients and collect informed consent (Additional file 5) and data. The project assistants will receive bedside and simulation training in all the included sample methods on how to obtain an optimal specimen. Furthermore, a guideline on how to perform the expiratory technique will be developed to support consistent data collection.

\section{Trial population}

\section{Screening data $\{21\}$}

Screenings data from previous studies was used to estimate the sample size of our trial population $[9,10$, $23,27,28]$. Use of this data ensures our results will be reproducible.

\section{Eligibility \{22\}}

Adults admitted to the ED with suspected LRTI will be invited to participate in the study if the attending physician identifies one of the following pulmonary symptoms: dyspnea, cough, expectoration, chest pain, or fever (Fig. 1).

Patients will be excluded if the following occur:

- The attending physician finds that participation will delay urgent treatment

- Patients are transferred to an intensive care unit

- Patients have severe immunodeficiencies such as human immunodeficiency virus infection (a CD4

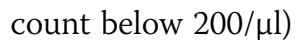

- Patients are treated with immunosuppressive therapy such as chemo- or radiotherapy

- Patients are treated with steroids in a prednisolone equivalent dosage of more than $20 \mathrm{mg} /$ day for 2 weeks up to the current admission or if consent cannot be obtained 


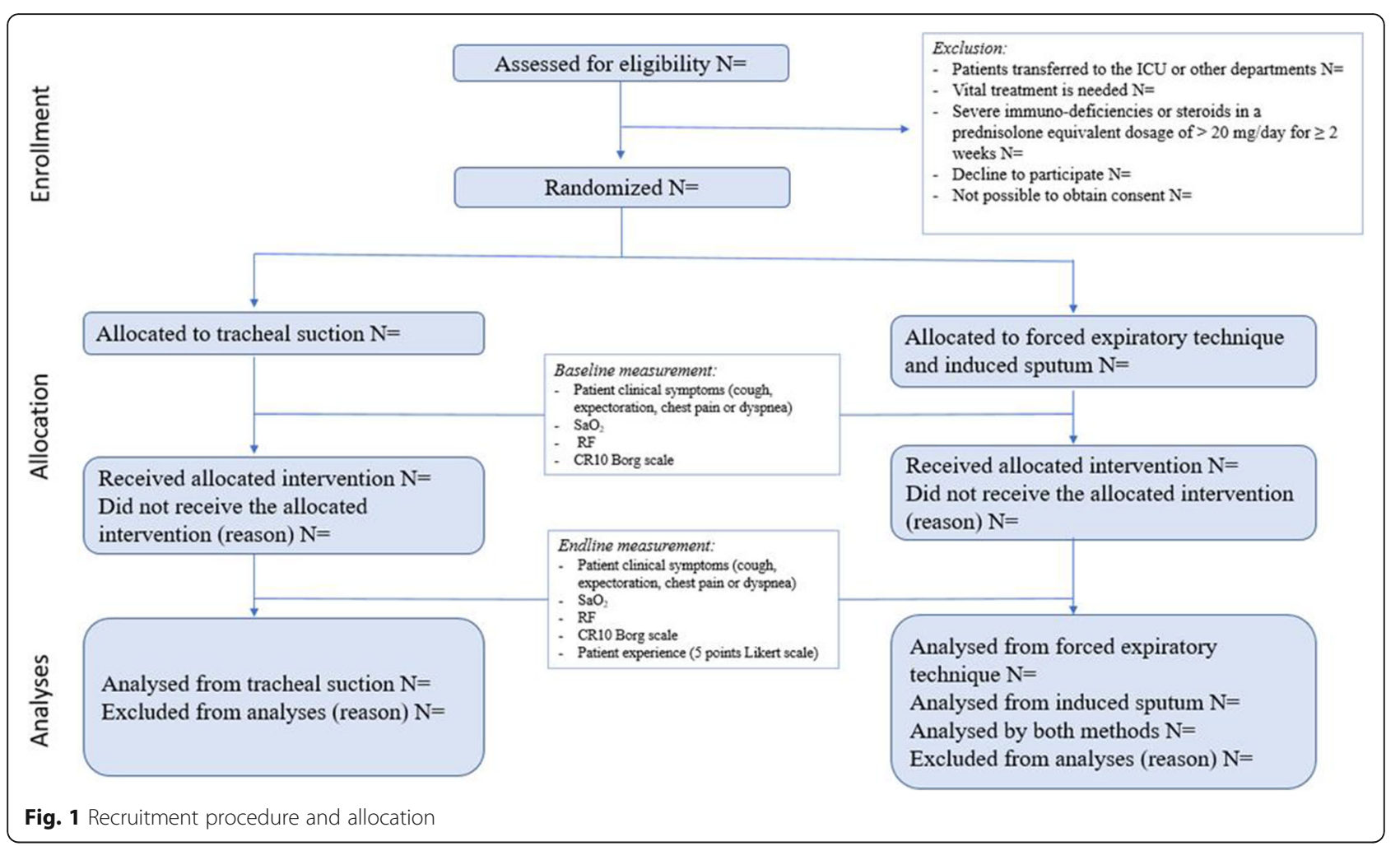

\section{Recruitment $\{23\}$}

Admitted patients with suspected LRTI will be identified in the patient management system (CETREA 4.2.0.0.) at the ED by a project assistant. The attending physician will confirm eligibility, and the patient's verbal and written consent will be obtained by the project assistant within the first hour of admission.

\section{Randomization $\{10\}$}

Within an hour after recruitment, enrolled patients are randomly assigned to either control (sputum collection by tracheal suction) or intervention (sputum collection by forced expiratory techniques). Patients in the intervention group who cannot deliver a specimen will undergo tracheal suction according to regional guidelines, and these samples will not be included in the primary analyses.

Patients are allocated 1:1 according to a computergenerated randomization sequence without stratification using blocks of six programmed by an independent data manager, so the treatment assignment is made by chance and the allocation is completely independent of any prior treatment allocation to a patient. The randomization tool in Research Electronic Data Capture (REDCap) will be used [29, 30]. The project assistants administering the randomization will not have access to the randomization code and will be blinded to the randomization procedure, block sizes, and randomization sequence at all times during the trial period. In addition, allocation concealment is ensured, as the randomization is performed electronically, is not revealed before consent is obtained, and occurs immediately prior to sputum collection.

\section{Blinding}

Participants, project assistants, and outcome adjudicators are not blinded to the randomization as it is not possible. The investigator will be blinded until data analysis is completed.

\section{Exposure variables Tracheal suction}

Before tracheal suction is performed, the patient will be informed in detail about the procedure. The patient is introduced to Fowler's position (semi-sitting position in $45-60^{\circ}$ with knees either bent or straight) and encouraged to clear their airway with a deep cough. The catheter (EXTRUDAN Surgery Aps, Denmark, CH12, $530 \mathrm{~mm}$ ) tip is lubricated with Xylocaine (lidocaine $\mathrm{HCl}$ ) $2 \%$ jelly and inserted into the nares during inhalation and gently advanced about $40 \mathrm{~cm}$ without applying suction. The suction catheter port is covered, and suctioning is performed before withdrawing the catheter. Suction is set at $200-400 \mathrm{mmHg}$ negative pressure. If 
necessary, the specimen is diluted in sterile saline water before storage in a sterile container.

\section{Sputum samples collected by forced expiratory techniques}

These techniques are based on the patients' own attempts to deliver a sputum sample. The patient is brought into a $90^{\circ}$ sitting position and instructed to clear the mouth with water to minimize oropharyngeal contamination, and proper forced exhalation and the coughing technique are thoroughly explained [24, 31].

The patient takes 3 to 5 deep diaphragmatic breaths, inhaling through the nose, exhaling through pursed lips. The patient takes a normal breath and then squeezes it out by contracting the abdominal and chest wall muscles, with the mouth and glottis open, while whispering and forcing the word "huff" during exhalation. The procedure is repeated 3 to 5 times. As secretions enter the larger airways, the patient exhales from high-to-mid lung volume to clear secretions from more proximal airways. The procedure continues as the patient takes 3 to 5 relaxed diaphragmatic breaths before coughing again [24, 31]. The sputum sample is stored in a sterile container.

\section{Induced sputum}

Isotonic saline inhalation $(0,9 \%)$ is used to loosen and induce the sputum. It is given for $10 \mathrm{~min}$ with a nebulizer system (Unomedical Opti-Mist TM,2.1m, ref. 93-772mm) [22]. After that, a forced expiratory technique is performed, and the sputum sample is stored in a sterile container.

Inhalation medicine is permitted as part of concomitant care.

\section{Microbiological variables Microscopy}

Part of the sputum is placed on a microscope slide with a cotton swab, and a second microscope slide is used to distribute the material on the surface. The smear is then heat-fixed and Gram stained. The number of squamous epithelial cells and polymorphonuclear leucocytes per field of view (100× magnification) is registered. Samples with $<10$ squamous epithelial cells per field of view are classified as suitable (good quality), samples with $\geq 10$ squamous epithelial cells are classified as unsuitable (poor quality) [32]. The microscopy findings and classification are registered in the microbiological laboratory information system (MADS, Aarhus University Hospital, Aarhus, Denmark) and are accessible in the patient's file.

In addition, a different sputum quality assessment based on both squamous epithelial cells and polymorphonuclear leucocytes will be used in subanalyses. Samples with $<10$ squamous epithelial cells and $\geq 25$ polymorphonuclear leucocytes per field of view (100x magnification) are classified as suitable (good quality) [32].

The samples unable to be collected will be considered missing as the quality cannot be determined.

\section{Culture}

Sputum is transferred with a cotton swab to an agar plate with 5\% sheep blood (Beckton Dickinson) with a Staphylococcus aureus streak and a Chrom Orientation agar plate (Beckton Dickinson), and the inoculum is streaked over the agar surface. The blood agar is incubated at $35{ }^{\circ} \mathrm{C}$ in an atmosphere with $6 \% \mathrm{CO}_{2}$, and the Chrom-agar orientation is incubated at $35{ }^{\circ} \mathrm{C}$ in normal atmospheric conditions.

After 1-2 days of incubation, pathogens are identified and tested for antimicrobial susceptibility.

The pathogen identified by culture will be semiquantitatively assessed as numerous, some, or few, with a morphology description. Mixed flora judged to be pharyngeal flora will be registered as "pharyngeal flora". No growth of pathogens will be registered as "No growth of pathogens".

\section{Baseline variables $\{25\}$}

Demographics, symptoms, and smoking are collected from a patient interview. Vital parameters, comorbidities, suspicion of pneumonia, diagnostic packages [33], severe acute respiratory syndrome coronavirus 2 (SARS-CoV-2), disease severity assessment (CURB-65 [34], PSI [35], and Danish Emergency Process Triage (DEPT) [36]), blood tests (C-reactive protein, leucocytes, neutrophils) length of hospital stay, antibiotic consumption, and inhaled medicine are extracted from the patient's medical record (Table 1).

\section{Co-variables}

Co-variables (listed and defined below) will be measured and compared between the two allocation groups. Patients' symptoms, oxygen saturation, respiratory rate, and overall experience will be measured before (right after allocation) and after sputum collection (measured at the latest $10 \mathrm{~min}$ after the procedure). Side effects observed by the project assistants and experience by the patient during the procedure will be registered once after sputum collection. Data on mortality and readmission will be extracted from the patient medical record. The projects assistants will follow standardized protocols to register all the co-variables including the questions concerning patient experiences.

- Coughing, expectoration, dyspnea, and chest pain symptoms will be reported as binary variables. Aggravation of cough, expectoration, dyspnea, and chest pain experienced by the patient will be reported. 
Table 1 Demographic and baseline clinical characteristics

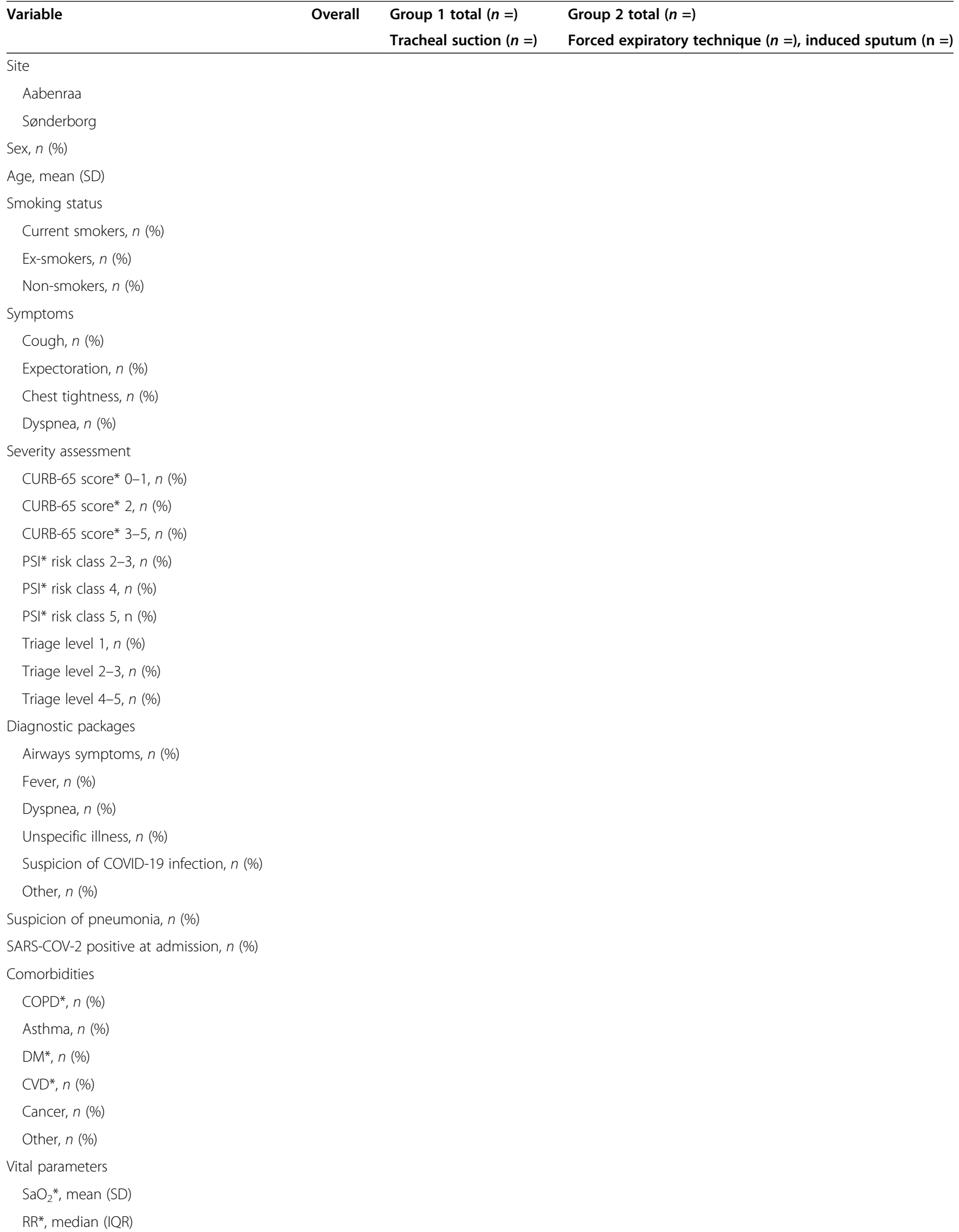


Table 1 Demographic and baseline clinical characteristics (Continued)

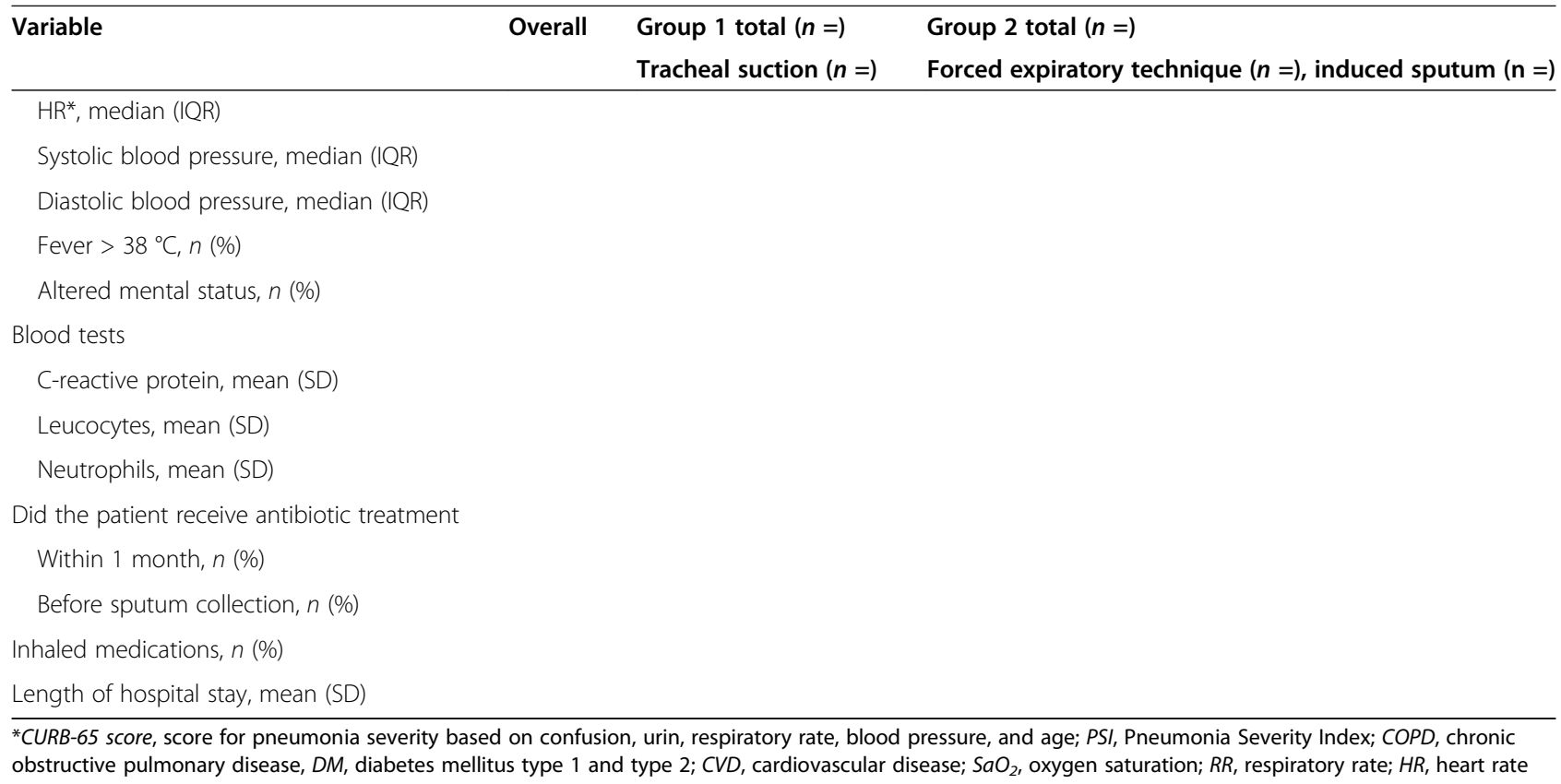

- Oxygen saturation. Oxygen saturation $\left(\mathrm{SaO}_{2}\right)$ will be measured using a pulse oximetry device to measure arterial oxygen saturation level in percentages up to $100 \%$. The acceptable $\mathrm{SaO}_{2}$ is $\geq 93 \%$. For patients with chronic obstructive disease, a $\mathrm{SaO}_{2}$ is acceptable at $\geq 88 \%$. $\mathrm{SaO}_{2}$ will be reported as mean (standard deviation (SD)) [37].

- Respiratory rate. The respiratory rate (RR) will be counted by the professional attending the patient. The respiratory rate is the number of breaths per minute that a patient takes while resting. It is assessed by counting the number of times the patient's chest rises in half a minute multiplied by two. RR will be reported as mean with standard deviation (SD). Acceptable results for RR are between 12 and 20 times per minute [38].

- Side effects observed by the project assistants. Project assistants will register any side effects such as bleeding from the airways and bronchospasm.

- Short-term mortality. Mortality within 7 days from admission to the emergency department

- Re-admission. Re-admission to hospital within 30 days of the original discharge

- Patient's overall experience of symptoms. Patient wellbeing and overall experience of symptoms will be measured at the latest $10 \mathrm{~min}$ after sputum collection. Patients will asked to verbally score their overall experience of their symptoms based on the CR10 Borg scale [39, 40]. The CR10 Borg scale assess the experience of patient symptoms ranges from " $0=$ nothing at all" to " 10 very very strong" and over 10 as "maximal" [40]. A visual support tool describing this scoring system will be used to assist the patient and the ordinal result will be reported as mean and SD.

- Patient's experience of the procedure to collect sputum sample: This will be measured once after an attempt at sputum collection. Patients will be asked to give a verbal score to the question: "What was your experience of this procedure?" using a fivepoint Likert scale. This scale ranges from "very bad, bad, neither bad nor good, good, very good". A visual support tool describing this scoring system will be used to assist the patient. The result will be reported in percentages. The development and validation of this scale was based on individual and focus group interviews using cognitive interview methods [41].

\section{Outcome definitions $\{26\}$}

1. Primary outcome. The quality of sputum samples, binary outcome

2. Secondary outcome 1. Number of adverse events, discrete outcome

(a) Aggravation of $\mathrm{SaO}_{2}$ and $\mathrm{SaO}_{2} \leq 93 \%$ (COPD patients $\leq 88 \%$ ), binary

(b) Aggravation of RR and RR lower than 12 or higher than 20 times per minute, binary

(c) Occurrence or aggravation of symptoms (cough, expectoration, dyspnea, chest pain), binary 
(d) Aggravation of patient symptoms experience, binary

(e) Occurrence of side effects observed by the project assistant, binary

(f) Death within 7 days from admission, binary

(g) 30 days re-admission after current hospitalization, binary

3. Secondary outcome 2. Patient experience of the sputum collection procedure, ordinal outcome

\section{Harms $\{30\}$}

The project includes collecting methods that are already applied and recommended in clinical practice. There are minimal indications of adverse effects of a spontaneous cough or induced sputum after inhaling an isotonic saline solution $(0.9 \%)[21,22]$. It is known that the tracheal suction routine carries a risk of airway bleeding, although this is rare and often short-lived [20]. Moreover, there may be short-term discomfort, especially during the tracheal suction procedure [19]. Patients will be informed of the adverse events verbally and in writing. In case of an aggravation of symptoms, the procedure that is being performed will be interrupted preventing patient participation. The procedures are known to have minimal risks and the hospital will provide routine ancillary post-trial care, if necessary.

\section{Data management $\{32\}$}

Data management plan version 1.0 is attached to the Additional file 4.

\section{Data monitoring}

During the data collection, an extern assessor will supervise the performance of all project assistants and an independent microbiologist expert will ensure data quality and continue registration of the specimens. The daily inclusion of participants will be monitored by the project investigator and discussed with the study assistants and steering committee.

After primary analysis of data, the results will be discussed and evaluated first in the steering committee and afterwards with all involved hospital departments.

\section{Statistical method}

\section{Sample size consideration $\{11\}$}

It is hypothesized that the intervention group receiving expiratory techniques is non-inferior to the tracheal suction group. Grouping two methods in the intervention arm, there will be fewer missing values and only two comparison groups.

Based on clinical practice and literature, it is assumed that no sample will be obtained from $50 \%$ of patients by forced expiratory technique alone $[9,10,42], 30 \%$ from patients by sputum induction combined with the forced expiratory technique $[23,43]$, and $10 \%$ from patients using tracheal suction [28]. The proportion of obtained samples classified as good quality is assumed to be $75 \%$ for the procedures using expiratory techniques [23, 27, 43] and 90\% for tracheal suction [28].

Considering these assumptions, the study's power is $84 \%$ with 260 patients, including missing values. The power of the study was calculated with a Monte Carlo simulation using logistic regression.

An internal register showed that at least 1250 patients were admitted to the study ED with suspicion of infection annually, and of these, 600 patients were diagnosed with pneumonia. Including 260 patients with suspected LRTI will take five months, taking into account exclusion criteria, weekends/holidays, and missing data.

To avoid overfitting in the primary analysis, we followed the relaxed one in ten rule for the fixed effects and one in 20 rule for the random effects [44].

\section{Statistics principles \\ Statistical interim analyses and stopping guidance $\{13\}$ \\ No interim analysis will be conducted.}

\section{Timing of final analyses $\{14+15\}$}

The final analyses will be conducted within three months of completion of inclusion, estimated to be ultimo September 2021. All outcomes will be analyzed collectively.

\section{Confidence interval and $p$-value $\{16+17+18\}$}

For the main analysis and the supplementary analysis with non-ordinal outcomes, 95\% confidence intervals will be reported. For the supplementary analysis of the secondary outcomes, bootstrapped confidence intervals will be calculated for ordinal outcomes to accommodate lack of fit. A $p$-value less than 0.05 will be considered statistically significant.

No adjusting for multiple testing will be utilized in any analyses, as we only have one primary outcome [45].

\section{Definition of analyses population \{20\}}

The primary analysis will be conducted according to the intention-to-treat (ITT) principle [46, 47].

We define the ITT population as all admitted patients suspected of LRTI who sign informed consent and subsequently are allocate to one of two sputum collection groups. Sputum samples obtained by tracheal suction from patients in the intervention group, who are not able to expectorate, will be analyzed separately from the ITT analyses.

The primary analysis compares the tracheal suction group and the expiratory technique group to identify the most effective method for obtaining good quality 
sputum samples. The secondary analysis consists of an agreement analysis [48] between forced expiratory technique alone and forced expiratory technique after sputum induction.

Additionally, analysis of the secondary outcome of adverse effects will be conducted at baseline (right after allocation) and at the latest, $10 \mathrm{~min}$. after the intervention. Clinical symptoms (cough, expectoration, chest pain, or dyspnea), $\mathrm{SaO}_{2}, \mathrm{RR}$, and CR10 Borg scale [40] will be measured. Furthermore, a 5-point Likert scale will be used to measure patient experience of the procedure. The analyses will be adjusted for variables with a large influence on the outcome, which is an odds ratio larger than 2, to accommodate bias [49].

Descriptive analyses will be performed on the pathogens found when the specimens are cultured according to differential quality criteria.

\section{Statistical analysis $\{27\}$}

\section{Descriptive statistics}

Descriptive statistics will be utilized to assess if exchangeability is fulfilled for baseline variables. For categorical variables, Fischer's exact test or chi-square test will be used to test if the distribution between the two groups is different. For discrete and continuous variables, the Wilcoxon rank-sum test or Student $t$-test will be utilized to assess if the distribution is similar between the two groups depending on normal data distribution.

\section{Analysis for primary outcome}

For the primary outcome, the following analyses will be utilized:

1. To assess the suitability of the sputum sample between the two collecting methods groups, a hierarchical mixed effect logistic model with and without imputed values will be utilized to accommodate the random effect's hierarchical structure, which manifests according to different personnel collecting the samples and geographical variation (Table 2).

2. Sub-analyses

(a) To assess agreement between forced expiratory technique alone and induced sputum combined with the forced expiratory technique, a kappa statistic will be calculated [48].

(b) If the kappa statistic indicates a lack of agreement between forced expiratory techniques [50], a sensitivity analysis comparing forced expiratory technique with tracheal suction and induced sputum with tracheal suction will be utilized. A hierarchical mixed effect logistic model will be utilized for both analyses.

(c) For patients where expectorate cannot be obtained in the intervention group, descriptive analyses will be conducted on the numbers of tracheal suctions performed and the obtained specimen quality.

(d) Kappa statistics will be performed to identify differential quality criteria of Gram stain results classified in three groups (Table 3). Bacterial pathogens are described in Table 4.

\section{Analysis for secondary outcomes}

For the secondary outcomes regarding adverse events and patient experience, the following analyses will be utilized (Table 2):

1. To investigate if the number of adverse events differs between groups, a mixed effect Poisson model will be utilized with and without imputed values to accommodate the random effect's hierarchical structure, which manifests according to different personnel collecting the samples and geographical variation.

(a) A sensitivity analysis for each adverse event type by either a chi-square test or Fischer's exact test will be performed (Table 5).

2. For the procedure experience using a five-point Likert scale, a Wilcoxon rank test will be performed to compare the two allocation groups according to sample methods.

Model control for the generalized linear mixed models will consist of assessing the deviance residuals' normality assumptions for each center by way of quantile-quantile plots. To assess if the variables in the descriptive analysis

Table 2 Results of the primary outcome regarding specimens' suitability and secondary outcome of adverse effects and patient experience. Tracheal suction method (reference group) compared to expiratory technique methods

\begin{tabular}{llc}
\hline Outcome & Unadjusted OR (Cl) $P$ & Adjusted OR (Cl) $P$ \\
\hline Quality assessment of the specimens & & Adjusted Coef (CI) $P$ \\
Adverse effects & Unadjusted Coef (Cl) $P$ & \\
Patient experience & & \\
Five-point Likert scale & & \\
\hline
\end{tabular}


Table 3 Gram stain results classified according to the number of epithelial cells and polymorphonuclear leucocytes judged by microscopy (100× magnification)

\begin{tabular}{lll}
\hline $\begin{array}{l}\text { Epithelial } \\
\text { cells }\end{array}$ & \multicolumn{2}{l}{ Polymorphonuclear leucocytes } \\
\cline { 2 - 3 } & $<\mathbf{1 0} 10-24$ & $\geq 25$ \\
\hline$\geq 25$ & & \\
$10-24$ & & \\
$<10$ & &
\end{tabular}

are normally distributed, quantile-quantile plots will be utilized.

If any of the generalized linear mixed models do not converge, a simplification of the covariance structure will be utilized.

\section{Withdrawal from the study $\{24\}$}

It is assumed that there will be minimal patient withdrawal from the study, as the specimens will be collected immediately after patient consent and allocation, and there will be no further patient contact. Withdrawals will be assumed to be missing completely at random (MCAR) [51].

\section{Missing data $\{28+29\}$}

Missing data includes patient withdrawals and sputum samples that are not possible to collect due to patients' nonproductive cough or noncompliance. Multiple imputations and complete case analysis will be conducted to investigate the influence of missing data on the results [52]. The guidelines concerning missing data suggested by Sterne et al. will be followed to ensure transparency [53].

Statistical software $\{31\}$

Statistical analyses will be performed using STATA version 17.0.

\section{Planned figures and tables}

The flow of participants will be illustrated in a flow diagram according to the CONSORT guidelines [54].

Baseline characteristics will be summarized in Table 1. Data will be presented as means with SD when normally distributed or as a median with interquartile range if the data is skewed. Dichotomous and categorical variables will be presented with number and percentage.

\section{Discussion}

The results of this study will provide important evidence on the optimal routine for obtaining high-quality sputum samples. This knowledge is essential when aiming to improve the initial management of patients admitted with suspected LRTI by supporting clinical decisions and targeted treatment.

The study will run in a real-life setting to increase the feasibility in implementing the methods afterwards.

This pre-defined SAP is essential to increase the study's transparency and explicitly describe protocol deviations to increase reproducibility, avoid any risk of reporting bias or data-driven analysis.

\section{Adherence and protocol deviations $\{19\}$}

Adjustments after the study start and before recruitment completion

Due to the SARS-CoV-2 pandemic, patient distribution was altered, and it was necessary to extend the recruitment to include the second site. The study period was adjusted with study start from October 19th to November 9th, and study completion from March 1st to July 5 th and will include at least 260 participants. New sample size and power calculations were performed where the clinical significant absolute difference was set at $15 \%$ points. The personnel conducting the sample size calculations do not know the participants' outcomes.

Table 4 Bacterial pathogens identified in culture according to differential quality criteria. Results are presented in percentages

\begin{tabular}{|c|c|c|c|}
\hline & $\begin{array}{l}\text { Squamous cells } \\
<10\end{array}$ & $\begin{array}{l}\text { Squamous cells } \\
10-24\end{array}$ & $\begin{array}{l}\text { Squamous cells } \\
\geq 25\end{array}$ \\
\hline \multicolumn{4}{|l|}{ Bacterial agents } \\
\hline \multicolumn{4}{|l|}{ Streptococcus pneumonia } \\
\hline \multicolumn{4}{|l|}{ Haemophilus influenza } \\
\hline \multicolumn{4}{|l|}{ Moraxella catarrhalis } \\
\hline \multicolumn{4}{|l|}{ Pseudomonas aeruginosa } \\
\hline \multicolumn{4}{|l|}{ Staphylococcus aureus } \\
\hline \multicolumn{4}{|l|}{ Enterobacteriaceae } \\
\hline \multicolumn{4}{|l|}{ Hemolytic streptococci } \\
\hline \multicolumn{4}{|l|}{ Pharyngeal flora } \\
\hline \multicolumn{4}{|l|}{ No growth of pathogens } \\
\hline Others & & & \\
\hline
\end{tabular}


Table 5 Sensitivity analyses for adverse events

\begin{tabular}{l}
\hline Adverse events $\quad$ Expiratory techniques, $\boldsymbol{n}(\%)$ \\
\hline Vital parameters \\
$\mathrm{SaO}_{2}{ }^{\mathrm{a}}$ \\
$\mathrm{RR}^{\mathrm{a}}$ \\
$\mathrm{CR} 10$ Borg scale \\
Side effects \\
Bleeding \\
Bronchospasm $\boldsymbol{n}$ (\%) \\
Others \\
Patient symptoms \\
Cough \\
Dyspnea \\
Expectoration \\
Chest tightness
\end{tabular}

${ }^{\mathrm{a}} \mathrm{SaO}_{2}$, oxygen saturation; $R R$, respiratory rate

\section{Adjustments before study start but after study protocol acceptance}

The increased SARS-CoV-2 situation did not make it possible to investigate the washed sputum technique analyses as it requires training of the staff. Therefore, this analysis was withdrawn from the study. The quality criteria for all randomized patients will follow the standard used by the microbiological department to reduce missing data (sputum samples containing $<10$ squamous epithelial cells per low power field of view are registered as usable material). The quality criteria of squamous epithelial cell and polymorphonuclear leucocytes classified in groups of $<10,10-24, \geq 25$ per low power field of view, will be registered as intended when available, making possible secondary analyses on sputum suitability.

All patients included in the trial will be asked how they experienced the collected method based on a fivepoint Likert scale instrument developed before the study start.

Protocol amendments will be registered directly with ClinicalTrials.gov (NCT04595526) (Additional file 2) and ethics committees and described explicitly in future publications.

\section{Abbreviations}

ED: Emergency department; LRT: Lower respiratory tract; LRTI: Lower respiratory tract infection; OPEN: Open Patient data Explorative Network; REDCap: Research Electronic Data Capture; SAP: Statistical analyses plan

\section{Supplementary Information}

The online version contains supplementary material available at https://doi. org/10.1186/s13063-021-05639-1.

Additional file 1. Spirit schedule.

Additional file 2. World Health Organization trial registration data.
Additional file 3. Spirit checklist

Additional file 4. Data management plan.

Additional file 5. Informed consent.

Additional file 6. SAP guideline.

\section{Acknowledgements}

The authors thank the Microbiological Department Hospital of Southern Denmark and chief microbiologist Steen Lomborg Andersen for support during the study on quality assurance of microbiological analyses and instructions of laboratory technicians. The authors appreciate the data management support and advice provided and REDCap [29,30] hosted by OPEN, Open Patient data Explorative Network, Odense University Hospital, Region of Southern Denmark.

\section{Trial status}

SAP version 1.0 was developed on the 18th of December 2020. Protocol to Ethics committee version 4.0 was updated on the 27 th January 2021.

Recruitment starts 9th November 2020.

Recruitment is estimated to be completed on the 5th July 2021.

\section{Steering committee}

This committee is composed of representatives from the participating departments: emergency, microbiology, biochemistry and immunology, and research and learning. The committee's role is to develop the scientific framework of the study and make final decisions on major issues during data collection and the data management period. Members of the steering committee are MBC, HSA, TS, CBM, FSR, and AKP.

\section{Authors' contributions}

MBC, HSA, TS, CBM, and FSR were involved in the planning of the design and method of the study. MBC and AKP drafted the manuscript in the collaboration of HSA. AKP contributed substantially with the statistical plan. $\mathrm{MBC}$ is the study investigator and HAS the research chief. All authors contributed to scientific knowledge. The work was critically revised by HSS, CBM, FSR, and TS. The authors read and approved the final manuscript.

\section{Funding}

The University of Southern Denmark (Campusvej 55, 5230 Odense, Denmark; sdu@sdu.dk) is funding the salary of the principal investigator. The University Hospital of Southern Denmark (Kresten Philipsensvej 15, 6200 Aabenraa, Denmark; shs.kontakt@rsyd.dk ) will cover the costs related to the material required during the study. These financial sponsors have no influence on the data, analysis, results, or content of the publication. 


\section{Availability of data and materials}

Due to Danish laws on personal data, data cannot be shared publicly. The person responsible for the research is the principal investigator and corresponding author that together with the Department of Health Research and the University Hospital of Southern Denmark owns the data and has access to the final data-set. To request this data, please contact the corresponding author for more information. For ancillary studies, a new consent will need to be given by the Regional Committees on Health Research Ethics for Southern Denmark.

\section{Declarations}

Ethics approval and consent to participate

The project was approved by the Regional Committees on Health Research Ethics for Southern Denmark (S-20200133), registered by the Danish Data Protection Agency (20/41767) and by ClinicalTrials.gov (NCT04595526). Written informed consent will be obtained from the participants before participation in the study.

\section{Consent for publication}

Not applicable.

\section{Competing interests}

The authors declare that they have no competing interests.

\section{Author details}

${ }^{1}$ Emergency Department, University Hospital of Southern Denmark, Kresten Philipsens vej 15, 6200 Aabenraa, Denmark. ²Department of Regional Health Research, University of Southern Denmark, Aabenraa, Denmark. ${ }^{3}$ Department of Biochemistry and Immunology, University Hospital of Southern Denmark, Kresten Philipsens vej 15,6200 Aabenraa, Denmark. ${ }^{4}$ Department of Clinical Microbiology, Odense University Hospital, J.B. Winsløwsvej 21-24, 5000 Odense C, Denmark. ${ }^{5}$ Department of Research and Learning, University Hospital of Southern Denmark, Kresten Philipsens vej 15, 6200, Aabenraa, Denmark.

Received: 6 May 2021 Accepted: 15 September 2021

\section{Published online: 02 October 2021}

\section{References}

1. Gamble C, Krishan A, Stocken D, Lewis S, Juszczak E, Doré C, et al. Guidelines for the content of statistical analysis plans in clinical trials. Jama. 2017;318(23):2337-43. https://doi.org/10.1001/jama.2017.18556.

2. Chan A-W, Tetzlaff JM, Gotzsche PC, Altman DG, Mann H, Berlin JA, et al SPIRIT 2013 explanation and elaboration: guidance for protocols of clinical trials. Bmj. 2013;346(jan08 15). https://doi.org/10.1136/bmj.e7586.

3. Estimates of the global, regional, and national morbidity, mortality, and aetiologies of lower respiratory tract infections in 195 countries: a systematic analysis for the Global Burden of Disease Study 2015. Lancet Infect Dis, 2017. 17(11): p. 1133-1161, DOI: https://doi.org/10.1016/S1473-3 099(17)30396-1.

4. WHO. The top-10-causes-of-death: WHO; 2020 [updated 9 december 2020; cited 20219 september ]. Available from: https://www.who.int/news-room/ fact-sheets/detail/the-top-10-causes-of-death.

5. Welte T, Torres A, Nathwani D. Clinical and economic burden of community-acquired pneumonia among adults in Europe. Thorax. 2012; 67(1):71-9. https://doi.org/10.1136/thx.2009.129502

6. Boyanova L. Direct Gram staining and its various benefits in the diagnosis of bacterial infections. Postgraduate medicine. 2018;130(1):105-10. https://doi. org/10.1080/00325481.2018.1398049.

7. Ogawa H, Kitsios GD, Iwata M, Terasawa T. Sputum Gram stain for bacterial pathogen diagnosis in community-acquired pneumonia: a systematic review and bayesian meta-analysis of diagnostic accuracy and yield. Clin Infect Dis. 2020;71(3):499-513. https://doi.org/10.1093/cid/ciz876.

8. Ewig S, Schlochtermeier M, Goïke N, Niederman MS. Applying sputum as a diagnostic tool in pneumonia: limited yield, minimal impact on treatment decisions. Chest. 2002;121(5):1486-92. https://doi.org/10.1378/chest.121.5.14 86.

9. Cartuliares MB, et al. Limited value of sputum culture to guide antibiotic treatment in a Danish emergency department. Dan Med J. 2020;67(11): A11190641.
10. Garcia-Vazquez E, et al. Assessment of the usefulness of sputum culture for diagnosis of community-acquired pneumonia using the PORT predictive scoring system. Archives of Internal Medicine. 2004;164(16):1807-11. https:// doi.org/10.1001/archinte.164.16.1807.

11. Metlay JP, Waterer GW, Long AC, Anzueto A, Brozek J, Crothers K, et al. Diagnosis and treatment of adults with community-acquired pneumonia. An official clinical practice guideline of the American Thoracic Society and Infectious Diseases Society of America. American Journal of Respiratory and Critical Care Medicine. 2019;200(7):e45-67. https://doi.org/10.1164/rccm.201 908-1581ST.

12. The Region of Southern Denmark, t.r.a.w., Den Regionale Antibiotikagruppe. Diagnostik og behandling af akutte infektioner på sygehusene i Region Syddanmark. 2016.

13. Eccles $\mathrm{S}$, et al. Diagnosis and management of community and hospital acquired pneumonia in adults: summary of NICE guidance. BMJ. 2014;349: g6722.

14. Geckler RW, Gremillion DH, McAllister CK, Ellenbogen C. Microscopic and bacteriological comparison of paired sputa and transtracheal aspirates. J Clin Microbiol. 1977:6(4):396-9. https://doi.org/10.1128/jcm.6.4.396-399.1977.

15. Nagendra S, Bourbeau P, Brecher S, Dunne M, LaRocco M, Doern G. Sampling variability in the microbiological evaluation of expectorated sputa and endotracheal aspirates. J Clin Microbiol. 2001;39(6):2344-7. https://doi. org/10.1128/JCM.39.6.2344-2347.2001.

16. Tetenta S, Metersky ML. Tracheal aspirate Gram stain has limited sensitivity and specificity for detecting Staphylococcus aureus. Respirology. 2011;16(1): 86-9. https://doi.org/10.1111/j.1440-1843.2010.01855.x.

17. Irwin RS, Demers RR, Pratter MR, Erickson AD, Farrugia R, Teplitz C. Evaluation of methylene blue and squamous epithelial cells as oropharyngeal markers: a means of identifying oropharyngeal contamination during transtracheal aspiration. J Infect Dis. 1980;141(2):16571. https://doi.org/10.1093/infdis/141.2.165.

18. Overend TJ, Anderson CM, Brooks D, Cicutto L, Keim M, McAuslan D, et al. Updating the evidence base for suctioning adult patients: a systematic review. Canadian respiratory journal. 2009;16(3):e6-e17. https:/doi.org/10.1155/2009/872921.

19. Arroyo-Novoa CM, Figueroa-Ramos MI, Puntillo KA, Stanik-Hutt J, Thompson $\mathrm{CL}$, White $\mathrm{C}$, et al. Pain related to tracheal suctioning in awake acutely and critically ill adults: a descriptive study. Intensive Crit Care Nurs. 2008;24(1): 20-7. https://doi.org/10.1016/j.iccn.2007.05.002.

20. Van de Leur JP, et al. Endotracheal suctioning versus minimally invasive airway suctioning in intubated patients: a prospective randomised controlled trial. Intensive Care Med. 2003;29(3):426-32. https://doi.org/10.1 007/s00134-003-1639-9.

21. Pizzichini $M$, et al. Safety of sputum induction. European Respiratory Journal. 2002;20(37 suppl):9s-18s.

22. Chanez $P$, et al. Sputum induction. European Respiratory Journal. 2002;20(37 suppl):3s-8s

23. Bandyopadhyay T, Gerardi DA, Metersky ML. A comparison of induced and expectorated sputum for the microbiological diagnosis of community acquired pneumonia. Respiration. 2000;67(2):173-6. https:/doi.org/10.1159/000029482

24. Lewis LK, Williams MT, Olds TS. The active cycle of breathing technique: a systematic review and meta-analysis. Respir Med. 2012;106(2):155-72. https://doi.org/10.1016/j.rmed.2011.10.014.

25. Ferreira ACM, Marson FAL, Cohen MA, Bertuzzo CS, Levy CE, Ribeiro AF, et al. Hypertonic saline as a useful tool for sputum induction and pathogen detection in cystic fibrosis. Lung. 2017;195(4):431-9. https://doi.org/10.1007/ s00408-017-0008-3.

26. Schulz KF, Altman DG, Moher D, for the CONSORT Group. CONSORT 2010 statement: updated guidelines for reporting parallel group randomised trials. BMJ. 2010;340(mar23 1):c332. https://doi.org/10.1136/bmj.c332.

27. Chuard C, Fracheboud D, Regamey C. Effect of sputum induction by hypertonic saline on specimen quality. Diagn Microbiol Infect Dis. 2001; 39(4):211-4. https://doi.org/10.1016/S0732-8893(01)00231-0.

28. Bartlett JG. Diagnostic accuracy of transtracheal aspiration bacteriologic studies. Am Rev Respir Dis. 1977;115(5):777-82. https://doi.org/10.1164/a rrd.1977.115.5.777

29. Harris PA, Taylor R, Thielke R, Payne J, Gonzalez N, Conde JG. Research electronic data capture (REDCap) — a metadata-driven methodology and workflow process for providing translational research informatics support. Journal of biomedical informatics. 2009;42(2):377-81. https://doi.org/10.101 6/j.jbi.2008.08.010. 
30. Harris PA, et al. The REDCap consortium: building an international community of software platform partners. J Biomed Inform. 2019;95:103208. https://doi.org/10.1016/j.jbi.2019.103208.

31. Fink JB. Forced expiratory technique, directed cough, and autogenic drainage. Respiratory Care. 2007;52(9):1210-23.

32. Murray PR, Washington JA. Microscopic and baceriologic analysis of expectorated sputum. Mayo Clin Proc. 1975;50(6):339-44.

33. Nørgaard B, et al. Diagnostic packages can be assigned accurately in emergency departments. A multi-centre cohort study. Dan Med J. 2016; 63(6):A5240.

34. Lim WS, van der Eerden M, Laing R, Boersma WG, Karalus N, Town Gl, et al. Defining community acquired pneumonia severity on presentation to hospital: an international derivation and validation study. Thorax. 2003;58(5): 377-82. https://doi.org/10.1136/thorax.58.5.377.

35. Fine MJ, Auble TE, Yealy DM, Hanusa BH, Weissfeld LA, Singer DE, et al. A prediction rule to identify low-risk patients with community-acquired pneumonia. N Engl J Med. 1997;336(4):243-50. https://doi.org/10.1056/ NEJM199701233360402.

36. Iversen AKS, Kristensen M, Østervig RM, Køber L, Sölétormos G, Lundager Forberg J, et al. A simple clinical assessment is superior to systematic triage in prediction of mortality in the emergency department. Emerg Med J. 2019;36(2):66-71. https://doi.org/10.1136/emermed-2016-206382.

37. O'Driscoll BR, Howard LS, Davison AG. BTS guideline for emergency oxygen use in adult patients. Thorax. 2008;63(Suppl 6):vi1-68.

38. Olsson T, Terent A, Lind L. Rapid Emergency Medicine score: a new prognostic tool for in-hospital mortality in nonsurgical emergency department patients. J Intern Med. 2004;255(5):579-87. https://doi.org/1 0.1111/j.1365-2796.2004.01321.x.

39. Ohbayashi H. Comparison of the rapid effects of single inhalations of formoterol and tiotropium bromide on respiratory function and COPD symptoms in a randomized crossover study. Respir Investig. 2017;55(6):34856. https://doi.org/10.1016/j.resinv.2017.07.004

40. Borg GA. Psychophysical bases of perceived exertion. Med Sci Sports Exerc. 1982;14(5):377-81. https://doi.org/10.1249/00005768-198205000-00012.

41. Patrick DL, Burke LB, Gwaltney CJ, Leidy NK, Martin ML, Molsen E, et al. Content validity--establishing and reporting the evidence in newly developed patient-reported outcomes (PRO) instruments for medical product evaluation: ISPOR PRO good research practices task force report: part 1--eliciting concepts for a new PRO instrument. Value Health. 2011; 14(8):967-77. https://doi.org/10.1016/j.jval.2011.06.014.

42. Shariatzadeh MR, Marrie TJ. Does sputum culture affect the management and/or outcome of community-acquired pneumonia? East Mediterr Health J. 2009;15(4):792-9. https://doi.org/10.26719/2009.15.4.792.

43. Guiot J, Demarche S, Henket M, Paulus V, Graff S, Schleich F, et al. Methodology for sputum induction and laboratory processing. J Vis Exp. 2017;130(130). https://doi.org/10.3791/56612

44. Vittinghoff $E$, McCulloch CE. Relaxing the rule of ten events per variable in logistic and Cox regression. Am J Epidemiol. 2007;165(6):710-8. https://doi. org/10.1093/aje/kwk052.

45. Bender R, Lange S. Adjusting for multiple testing - when and how? Journal of clinical epidemiology. 2001;54(4):343-9. https://doi.org/10.1016/S0895-43 56(00)00314-0

46. White, I.R., Horton N.J., Carpenter J., statistics ....., Pocock S.J., Strategy for intention to treat analysis in randomised trials with missing outcome data. Bmj, 2011. 342: p. d40, feb07 1, DOl: https://doi.org/10.1136/bmj.d40.

47. Heritier SR, Gebski VJ, Keech AC. Inclusion of patients in clinical trial analysis: the intention-to-treat principle. Medical Journal of Australia. 2003;179(8): 438-40. https://doi.org/10.5694/j.1326-5377.2003.tb05627.x.

48. de Vet $\mathrm{HC}$, et al. When to use agreement versus reliability measures. J Clin Epidemiol. 2006;59(10):1033-9. https://doi.org/10.1016/j.jclinepi.2005.10.015.

49. Gail MH, Wieand S, Piantadosi S. Biased estimates of treatment effect in randomized experiments with nonlinear regressions and omitted covariates. Biometrika. 1984;71(3):431-44. https://doi.org/10.1093/biomet/71.3.431.

50. Cohen J. A coefficient of agreement for nominal scales. Educational and psychological measurement. 1960;20(1):37-46. https://doi.org/10.1177/00131 6446002000104

51. Seaman $\mathrm{S}$, et al. What is meant by "missing at random"? Statistical Science. 2013;28:257-68.

52. van der Heijden GJ, et al. Imputation of missing values is superior to complete case analysis and the missing-indicator method in multivariable diagnostic research: a clinical example. J Clin Epidemiol. 2006;59(10):1102-9. https://doi.org/10.1016/j.jclinepi.2006.01.015.

53. Sterne JA, et al. Multiple imputation for missing data in epidemiological and clinical research: potential and pitfalls. Bmj. 2009;338(jun29 1):b2393. https:// doi.org/10.1136/bmj.b2393.

54. Moher D, Hopewell S, Schulz KF, Montori V, Gotzsche PC, Devereaux PJ, et al. CONSORT 2010 explanation and elaboration: updated guidelines for reporting parallel group randomised trials. Bmj. 2010;340(mar23 1):c869. https://doi.org/10.1136/bmj.c869.

\section{Publisher's Note}

Springer Nature remains neutral with regard to jurisdictional claims in published maps and institutional affiliations.
Ready to submit your research? Choose BMC and benefit from:

- fast, convenient online submission

- thorough peer review by experienced researchers in your field

- rapid publication on acceptance

- support for research data, including large and complex data types

- gold Open Access which fosters wider collaboration and increased citations

- maximum visibility for your research: over $100 \mathrm{M}$ website views per year

At $\mathrm{BMC}$, research is always in progress.

Learn more biomedcentral.com/submissions 